\title{
Parameter Estimations for Skew Ornstein-Uhlenbeck Processes
}

\author{
Ailing Feng \\ School of Sciences, Hebei University of Technology, \\ School of Sciences, Hebei University of Technology, Beichen district,Tianjin 300401, China
}

\begin{abstract}
In this paper, we study the parameter estimations for the skew Ornstein-Uhlenbeck processes based on continuous observations. However, dealing with the skew Ornstein-Uhlenbeck processes are tough because of the appearance of the local time. Therefore, we transform the skew Ornstein-Uhlenbeck processes into the standard diffusion processes, and then utilize the measure transformation to obtain the log likelihood function. At the same time we derive the formulas for the estimators of the drift parameter. Furthermore, we prove their consistency and asymptotic normality.
\end{abstract}

Keywords: Parameter estimation, Skew Ornstein-Uhlenbeck process, The log likelihood function, Strong consistency, Asymptotic normality.

\section{Introduction}

Over the past few decades, many scholars have studied different problems for skew processes. The pioneering work by Itô and McKean [6], which investigated the skew Brownian motion as the sign reversing of excursions of the standard Brownian motion based on a certain probability. The path of a skew Brownian motion behaves like a normal Brownian motion until it hits level 0 . When arriving at 0 , it will move upwards or downwards with probability $\mathrm{p}$ and $1-\mathrm{p}$ respectively. In particular, when $\mathrm{p}=0$ or 1 , it becomes a reflected Brownian motion. For the deeper properties of skew Brownian motion, see Antoine Lejay [9]. Now, the skew process has received a lot of attention since it has been found widespread use in a broad range of application domains, such as in physical, Lang [8]; biological, Cantrell and Cosner[3]; and mathematical finance models, Decamps et al. [4] and the reference therein.

In recent years, more and more new problems appear in our field of vision, among those generalizations, parameter estimation for the skew process has become an increasingly popular discussion. However, as far as we know, there is few literature to study this aspect. So our purpose is to find a method for estimating the drift parameters. Fortunately, as a special case, some significant development about the reflected Ornstein-Uhlenbeck process has been made recently, for instance, a sequential maximum likelihood estimator for the reflected Ornstein-Uhlenbeck process, we can see Lee et al. [10] and Lijun Bo and Xuewei Yang [2]. Then Yaozhong Hu et al. [5] considered a parameter estimation problem for a one-dimensional reflected Ornstein-Uhlenbeck with discrete observations.

The main contribution of this paper is to show that parameter estimations for skew Ornstein-Uhlenbeck processes. For the fist time, we introduce the skew Ornstein-Uhlenbeck process. Let $\left(\Omega, \mathrm{F},\left\{\mathrm{F}_{t}\right\}_{t \geq 0}, P\right)$ be a filtered probability space with the filtration $\left\{\mathrm{F}_{t}\right\}_{t \geq 0}$ satisfying the usual conditions. Suppose
$\left\{X_{t}: t \geq 0\right\}$ is a skew Ornstein-Uhlenbeck process with the infinitesimal drift $k-\alpha x$ and infinitesimal variance $\sigma^{2}$ on this space. The process $X$ is defined to satisfy the following stochastic differential equation (SDE):

$\left\{\begin{array}{l}d X_{t}=\left(k-\alpha X_{t}\right) d t+\sigma d W_{t}+(2 p-1) d L_{t}, \\ X_{0}=0,\end{array}\right.$

Where $k \in R, \sigma, \alpha \in(0, \infty), 0 \leq p \leq 1, W_{t}$ is a one-dimensional standard Brownian motion on the probability space $\left(\Omega, \mathrm{F},\left\{\mathrm{F}_{t}\right\}_{t \geq 0}, P\right), \quad L_{t}$ is the symmetrical local time of $X_{t}$ at the skew level 0 , that is to say, the process $L_{t}$ increases only when $X$ is zero, so that $\int_{[0, \infty)} I\left(X_{t}>0\right) d L_{t}=0$,

where $I(\cdot)$ is the indicator function. More details on the skew Ornstein-Uhlenbeck process can be found Suxin Wang [13].

In this paper, we expand the previously known results on the parameter estimation problems for a reflected Ornstein-Uhlenbeck process in Lijun Bo [1]. However, parameter estimation for the skew Ornstein-Uhlenbeck process is in general more tractable. To overcome such limitations, we give a necessary transformation to simplify equation. Moreover, we get the log likelihood function by the measure transformation. Hence, for the sake of simplicity, we will assume $\sigma$ and $p$ are known in whole paper.

The remainder of this paper is organized as follows. Section two introduce some basic knowledge about seeking likelihood function. Section three shows that how can we obtain the log likelihood function through a series of transformations, and then we explicitly compute the estimators of the drift parameter. Section four provides the strong consistency and asymptotic normality.

\section{Preliminaries}

Volume 5 Issue 6, June 2016 www.ijsr.net 


\section{International Journal of Science and Research (IJSR) \\ ISSN (Online): 2319-7064}

Index Copernicus Value (2013): 6.14 | Impact Factor (2015): 6.391

In this section, we first introuce a special diffusion process, and then give a probability measure transform for the special diffusion process, which is useful for seeking a likelihood function in the coming section.

Supposing $X_{t}$ is a solution to the stochastic differential equation

$$
d X_{t}=a\left(t, X_{t}, \theta\right) d t+d W_{t}, \quad X_{0}=0, t \geq 0 。
$$

We assume that sufficient conditions hold on the coefficient $a(\cdot,, \theta)$ so that there exists a unique solution to equation (1) for any fixed $\theta \in \Theta$.

Firstly, we study a special diffusion process $X_{t}$ on the probability space $\left(\Omega, \mathrm{F},\left\{\mathrm{F}_{t}\right\}_{t \geq 0}, P\right)$,

$d X_{t}=d \bar{W}_{t}$,

Where $\bar{W}_{t}$ is a standard Browniam motion under the probability space $\left(\Omega, \mathrm{F},\left\{\mathrm{F}_{t}\right\}_{t \geq 0}, P\right)$.

Now, we define a new probability measure $R$ as follows: $\frac{d R}{d P}=\exp \left[\int_{0}^{T} a\left(t, X_{t}, \theta\right) d \bar{W}_{t}-\frac{1}{2} \int_{0}^{T} a^{2}\left(t, X_{t}, \theta\right) d t\right]$.

According to the measure transformation, it is straight forward to see that $W_{T}=-\int_{0}^{T} a\left(t, X_{t}, \theta\right) d t+\bar{W}_{T}$ is a standard Browniam motion under the measure $R$. As a consequence, $\left\{X_{t}: 0 \leq t \leq \infty\right\}$ is a diffusion process under the measure $R$, that is $d X_{t}=a\left(t, X_{t}, \theta\right) d+d W_{t}$.

Therefore, we can easily obtain the log likelihood function $\ell(\theta)=\log \frac{d R}{d P}=\int_{0}^{T} a\left(t, X_{t}, \theta\right) d X_{t}-\frac{1}{2} \int_{0}^{T} a^{2}\left(t, X_{t}, \theta\right) d t$

\section{The estimators of the skew Ornstein-Uhlenbeck process}

In this section, we discuss how to estimate the parameter of the skew Ornstein-Uhlenbeck process. However, due to the appearance of the local time, we deal with this problem is quite difficult, so we will remove the symmetric local time and get a piecewise tractable process. We can see that as a special cases as well when $p=0$ or $p=1$, the reflected Ornstein-Uhlenbeck process be mentioned in Lijun Bo [1], therefore, we just discuss $0<p<1$. For the convenience of research, it is necessary to assume $\sigma$ and $p$ are known in the whole paper.

Dfeine

$G(x)=\left\{\begin{array}{l}p x, \quad x \leq 0, \\ (1-p) x, \quad x>0 .\end{array}\right.$

and
$g(x)=G^{\prime}(x)=\left\{\begin{array}{l}p, \quad x \leq 0, \\ 1-p, \quad x>0 .\end{array}\right.$

Thus, it seems clear that $G$ is a continuous function with the inverse function $H$ :

$H(x)=\left\{\begin{array}{l}\frac{1}{p}, \quad x \leq 0, \\ \frac{1}{1-p}, \quad x>0 .\end{array}\right.$

Since $G(x)$ is the difference of convex functions, applying the generalized Itô formula (see e.g., Protter [11]) to $R_{t}=G\left(X_{t}\right)$, we can obtain

$$
\begin{gathered}
R_{t}=G\left(X_{0}\right)+\int_{0}^{t} G^{\prime}\left(X_{s}\right) d X_{s}+\frac{1}{2} \int_{0}^{t} G^{\prime \prime}\left(X_{s}\right) d\langle X\rangle_{s} \\
\quad+\frac{1}{2}\left[G^{\prime}(0+)-G^{\prime}(0-)\right] L_{t}^{X}(0) \\
=R_{0}+\int_{0}^{t} G^{\prime}\left(X_{s}\right)\left[\left(k-\alpha X_{s}\right) d s+\sigma d W_{s}\right. \\
\left.\quad+(2 p-1) d L_{s}^{X}(0)\right]+\frac{1}{2}(1-p-p) L_{t}^{X}(0) \\
=\int_{0}^{t} G^{\prime}\left(X_{s}\right)\left(k-\alpha X_{s}\right) d s+\int_{0}^{t} G^{\prime}\left(X_{s}\right) \sigma d W_{s} \\
+\int_{0}^{t} G^{\prime}\left(X_{s}\right)(2 p-1) d L_{s}^{X}(0)+\frac{1}{2}(1-2 p) L_{t}^{X}(0) \\
=\int_{0}^{t} G^{\prime}\left(X_{s}\right)\left(k-\alpha X_{s}\right) d s+\int_{0}^{t} G^{\prime}\left(X_{s}\right) \sigma d W_{s}
\end{gathered}
$$

where the last equality holds due to the fact:

$$
\begin{aligned}
\int_{0}^{t} G^{\prime}\left(X_{s}\right)(2 p-1) d L_{t}^{X}(0)+\frac{1}{2}(1-2 p) L_{t}^{X}(0) \\
=G^{\prime}(0)(2 p-1) L_{t}^{X}(0)+\frac{1}{2}(1-2 p) L_{t}^{X}(0) \\
=\frac{1}{2}\left[G^{\prime}(0+)+G^{\prime}(0-)\right](2 p-1) L_{t}^{X}(0) \\
\quad+\frac{1}{2}(1-2 p) L_{t}^{X}(0) \\
=\frac{1}{2}(1-p+p)(2 p-1) L_{t}^{X}(0)+\frac{1}{2}(1-2 p) L_{t}^{X}(0) \\
=0
\end{aligned}
$$

Then, we can derive that

$d R_{t}=G^{\prime}\left(X_{t}\right)\left(k-\alpha X_{t}\right) d t+G^{\prime}\left(X_{t}\right) \sigma d W_{t}$

$=g\left(H\left(R_{t}\right)\right)\left(k-\alpha H\left(R_{t}\right)\right) d t+g\left(H\left(R_{t}\right)\right) \sigma d W_{t}$

$=\left\{\begin{array}{l}p\left(k-\alpha H\left(R_{t}\right)\right) d t+\sigma p d W_{t}, \quad x \leq 0, \\ (1-p)\left(k-\alpha H\left(R_{t}\right)\right) d t+\sigma(1-p) d W_{t}, \quad x>0 .\end{array}\right.$

Hence it is necessary to discuss the parameter estimation of the drift parameter respectively. First of all, we show that the parameter estimation for skew Ornstein-Uhlenbeck when $x \leq 0$. That is to say,

$$
d R_{t}=p\left(k-\alpha H\left(R_{t}\right)\right) d t+\sigma p d W_{t}, \quad x \leq 0 .
$$

\section{Volume 5 Issue 6, June 2016}




\section{International Journal of Science and Research (IJSR) \\ ISSN (Online): 2319-7064}

Index Copernicus Value (2013): 6.14 | Impact Factor (2015): 6.391

Similarly to the conclusion mentioned above, we can define the log likelihood function under the measure $R$ as follows:

$$
\begin{aligned}
& \ell(k, \alpha) \\
= & \sigma^{2} p^{2} \log \frac{d R}{d P} \\
= & \sigma^{2} p^{2}\left[\int_{0}^{T} \frac{p\left(k-\alpha H\left(R_{t}\right)\right)}{\sigma p} d \bar{W}_{t}\right. \\
& \left.-\frac{1}{2} \int_{0}^{T}\left(\frac{p\left(k-\alpha H\left(R_{t}\right)\right)}{\sigma p}\right)^{2} d t\right] \\
= & \int_{0}^{T} p\left(k-\alpha H\left(R_{t}\right)\right) \sigma p d \bar{W}_{t} \\
& -\frac{1}{2} \int_{0}^{T}\left(p\left(k-\alpha H\left(R_{t}\right)\right)\right)^{2} d t \\
= & \int_{0}^{T} p\left(k-\alpha H\left(R_{t}\right)\right) d R_{t}-\frac{1}{2} \int_{0}^{T}\left[p^{2} k^{2}-2 \alpha p^{2} k H\left(R_{t}\right)\right. \\
& \left.+\alpha^{2} p^{2} H^{2}\left(R_{t}\right)\right] d t \\
= & p k R_{T}-\alpha p \int_{0}^{T} H\left(R_{t}\right) d R_{t}-\frac{1}{2} p^{2} k^{2} T \\
& +\alpha p^{2} k \int_{0}^{T} H\left(R_{t}\right) d t-\frac{1}{2} \alpha^{2} p^{2} \int_{0}^{T} H^{2}\left(R_{t}\right) d t .
\end{aligned}
$$

Through a simple derivation calculation, without any doubt the fact that

$$
\left\{\begin{array}{l}
\frac{\partial \ell(k, \alpha)}{\partial k}=p R_{T}-p^{2} k T+\alpha p^{2} \int_{0}^{T} H\left(R_{t}\right) d t=0 \\
\frac{\partial \ell(k, \alpha)}{\partial \alpha}=-p \int_{0}^{T} H\left(R_{t}\right) d R_{t}+k p^{2} \int_{0}^{T} H\left(R_{t}\right) d t \\
-\alpha p^{2} \int_{0}^{T} H^{2}\left(R_{t}\right) d t=0 .
\end{array}\right.
$$

With straightforward calculation, we get

$$
\left\{\begin{array}{l}
\hat{k}_{T}=\frac{\int_{0}^{T} H\left(R_{t}\right) d R_{t} \int_{0}^{T} H\left(R_{t}\right) d t-R_{T} \int_{0}^{T}\left(H\left(R_{t}\right)\right)^{2} d t}{p\left[\left(\int_{0}^{T} H\left(R_{t}\right) d t\right)^{2}-T \int_{0}^{T}\left(H\left(R_{t}\right)\right)^{2} d t\right]}, \\
\hat{\alpha}_{T}=\frac{T \int_{0}^{T} H\left(R_{t}\right) d R_{t}-R_{T} \int_{0}^{T} H\left(R_{t}\right) d t}{p\left[\left(\int_{0}^{T} H\left(R_{t}\right) d t\right)^{2}-T \int_{0}^{T}\left(H\left(R_{t}\right)\right)^{2} d t\right]} .
\end{array}\right.
$$

\section{The strong consistency and asymptotic normality}

In this section, we discuss the strong consistency and asymptotic normality of the parameter estimators.

Theorem 1. The estimator $\hat{k}_{T}$ of $k$ admits the strong consistency, i.e.

$\hat{k}_{T} \rightarrow k \quad$ a.s. $[R]$ as $T \rightarrow \infty$.

Proof: Because the range of the parameter $k$ is $(0, \infty)$. Then for any $\delta>0$ such that $k \pm \delta \in(0, \infty)$. According to the (3), we can obtain

$$
\begin{aligned}
& \ell(k \pm \delta, \alpha)-\ell(k, \alpha) \\
= & p(k \pm \delta) R_{T}-\alpha p \int_{0}^{T} H\left(R_{t}\right) d R_{t}-\frac{1}{2} p^{2}(k \pm \delta)^{2} T \\
& +\alpha p^{2}(k \pm \delta) \int_{0}^{T} H\left(R_{t}\right) d t-\frac{1}{2} \alpha^{2} p^{2} \int_{0}^{T}\left(H\left(R_{t}\right)\right) d t \\
& -\left[p k R_{T}^{2}-\alpha p \int_{0}^{T} H\left(R_{t}\right) d R_{t}-\frac{1}{2} p^{2} k^{2} T\right. \\
& \left.+\alpha p^{2} k \int_{0}^{T} H\left(R_{t}\right) d t-\frac{1}{2} \alpha^{2} p^{2} \int_{0}^{T}\left(H\left(R_{t}\right)\right) d t\right] \\
= & \pm p \delta R_{T}^{2} \mp p^{2} k \delta T-\frac{1}{2} p^{2} \delta^{2} T \pm \alpha p^{2} \delta \int_{0}^{T} H\left(R_{t}\right) d t \\
= & \pm p \delta\left[p \int_{0}^{T}\left(k-\alpha H\left(R_{t}\right)\right) d t+\sigma p d W_{t}\right] \mp p^{2} k \delta T \\
= & \pm \frac{1}{2} p^{2} \delta^{2} T \pm \alpha p^{2} \delta \int_{0}^{T} H\left(R_{t}\right) d t \\
& =\frac{1}{2} p^{2} \delta^{2} T \\
& \\
& \\
&
\end{aligned}
$$

Let $A_{t}= \pm p \delta W_{T}$ and $M_{T}=A_{t}^{2}$. Then we have

$$
\frac{\ell(k \pm \delta, \alpha)-\ell(k, \alpha)}{M_{T}}=\frac{ \pm p^{2} \sigma \delta W_{T}}{M_{T}}-\frac{1}{2} \text {. }
$$

We can see that, for each $\delta>0$,

$M_{T} \rightarrow \infty$ a.s.[R] as $T \rightarrow \infty$,

That is to say,

$$
\frac{ \pm p^{2} \sigma \delta W_{T}}{M_{T}} \rightarrow 0 \quad \text { a.s. }[R] \text { as } T \rightarrow \infty \text {. }
$$

According to the Lepingle' s law of large number (see Liptser and Shiryayev [7], Lemma 17.4, page 201), we can get $\frac{\ell(k \pm \delta, \alpha)-\ell(k, \alpha)}{M_{T}} \rightarrow-\frac{1}{2} \quad$ a.s. [R] as $T \rightarrow \infty$.

Similarly to the proof of Theorem 2.21 in Prakasa Rao [12], we can conclude that there is an $\hat{k}_{T} \in(k-\delta, k+\delta)$ such that $\ell^{\prime}\left(\hat{k}_{T}, \alpha\right)=0$ and $\hat{k}_{T} \rightarrow k \quad$ a.s. $[R]$ as $T \rightarrow \infty$.

Theorem 2. The estimator $\hat{\alpha}_{T}$ of $\alpha$ admits the strong consistency, i.e.

$\hat{\alpha}_{T} \rightarrow \alpha \quad$ a.s.[R] as $T \rightarrow \infty$.

Proof Similarly to the proof of the Theorem 1. because the range of the parameter $\alpha$ is $(0, \infty)$. Then for any $\delta>0$ such that $\alpha \pm \delta \in(0, \infty)$. we can have that

$$
\ell(k, \alpha \pm \delta)-\ell(k, \alpha)
$$




\section{International Journal of Science and Research (IJSR) \\ ISSN (Online): 2319-7064}

Index Copernicus Value (2013): 6.14 | Impact Factor (2015): 6.391

$$
\begin{aligned}
= & p k R_{T}-(\alpha \pm \delta) p \int_{0}^{T} H\left(R_{t}\right) d R_{t}-\frac{1}{2} p^{2} k^{2} T \\
& +(\alpha \pm \delta) p^{2} k \int_{0}^{T} H\left(R_{t}\right) d t \\
& -\frac{1}{2}(\alpha \pm \delta)^{2} p^{2} \int_{0}^{T}\left(H\left(R_{t}\right)\right)^{2} d t \\
& -\left[p k R_{T}-\alpha p \int_{0}^{T} H\left(R_{t}\right) d R_{t}-\frac{1}{2} p^{2} k^{2} T\right. \\
& \left.+\alpha p^{2} k \int_{0}^{T} H\left(R_{t}\right) d t-\frac{1}{2} \alpha^{2} p^{2} \int_{0}^{T}\left(H\left(R_{t}\right)\right)^{2} d t\right] \\
= & \mp p \delta \int_{0}^{T} H\left(R_{t}\right) d R_{t} \pm p^{2} \delta k \int_{0}^{T} H\left(R_{t}\right) d t \\
& \mp \alpha \delta p^{2} \int_{0}^{T}\left(H\left(R_{t}\right)\right) d t-\frac{1}{2} p^{2} \delta^{2} \int_{0}^{T}\left(H\left(R_{t}\right)\right)^{2} d t \\
= & \mp p \delta \int_{0}^{T} H\left(R_{t}\right)\left[p\left(k-\alpha H\left(R_{t}\right)\right) d t+\sigma p d W_{t}\right] \\
& \pm p^{2} \delta k \int_{0}^{T} H\left(R_{t}\right) d t \mp \alpha \delta p^{2} \int_{0}^{T}\left(H\left(R_{t}\right)\right)^{2} d t \\
& \mp \alpha \delta p^{2} \int_{0}^{T}\left(H\left(R_{t}\right)\right)^{2} d t-\frac{1}{2} \delta^{2} p^{2} \int_{0}^{T}\left(H\left(R_{t}\right)\right)^{2} d t \\
& -\frac{1}{2} \delta^{2} p^{2} \int_{0}^{T}\left(H\left(R_{t}\right)\right) d t \\
= & \mp p^{2} \delta k \int_{0}^{2} H \sigma \int_{0}^{T} H\left(R_{t}\right) d W_{t}-\frac{1}{2} p^{2} \delta^{2} \int_{0}^{T}\left(H\left(R_{t}\right)\right)^{2} d t \\
& \mp \sigma p^{2} \delta \int_{0}^{T} H\left(R_{t}\right) d W_{t} \pm p^{2} \delta \int_{0}^{T}\left(H\left(R_{t}\right)\right)^{2} d t \\
& \\
& \\
& \\
& \\
&
\end{aligned}
$$

Let $B_{t}= \pm \delta p H\left(R_{t}\right)$ and $N_{T}=B_{t}^{2}$. Then we have

$$
\frac{\ell(k \pm \delta, \alpha)-\ell(k, \alpha)}{N_{T}}=\frac{ \pm p^{2} \sigma \delta \int_{0}^{T} H\left(R_{t}\right) d W_{t}}{N_{T}}-\frac{1}{2} .
$$

If we can verified that, for each $\delta>0$,

$N_{T} \rightarrow \infty$ a.s.[R] as $T \rightarrow \infty$.

That is to say,

$$
\frac{ \pm p^{2} \sigma \delta \int_{0}^{T} H\left(R_{t}\right) d t}{N_{T}} \rightarrow 0 \quad \text { a.s. }[R] \text { as } T \rightarrow \infty .
$$

According to the Lepingle' s law of large number (see Liptser and Shiryayev [7], Lemma 17.4, page 201), we can get

$$
\frac{\ell(k \pm \delta, \alpha)-\ell(k, \alpha)}{N_{T}} \rightarrow-\frac{1}{2} \quad \text { a.s. }[R] \text { as } T \rightarrow \infty \text {. }
$$

Similarly to the proof of Theorem 2.21 in Prakasa Rao [12], we can conclude that there is an $\hat{\alpha}_{T} \in(\alpha-\delta, \alpha+\delta)$ such that $\ell^{\prime}\left(k, \hat{\alpha}_{T}\right)=0$ and $\hat{\alpha}_{T} \rightarrow \alpha \quad$ a.s. $[R]$ as $T \rightarrow \infty$.

Now it is sufficient to prove that (4) holds. It is known that the Ornstein-Uhlenbeck process

$d Z_{t}=p\left(k-\alpha Z_{t}\right) d t+\sigma p d W_{t}$

has the property $\int_{0}^{T} Z_{t}^{2} d t$ a.s.[R] as $T \rightarrow \infty$. On the other hand, recall $\left(R_{t}\right)_{t \geq 0}$ defined in (2). It is not hard to verify that $R_{t}-Z_{t}=e^{-\alpha t}\left(R_{0}-Z_{0}\right)$. It follows that if $R_{0} \geq Z_{0}$, for all $t \geq 0$. Thus we have $\int_{0}^{T} R_{t}^{2} d t \rightarrow \infty$ a.s. $[R]$ as $T \rightarrow \infty$, that is to say $\int_{0}^{T} H^{2}\left(R_{t}\right) d t \rightarrow \infty$ a.s.[R] as $T \rightarrow \infty$, which implies (4). The proof of the theorem is completed.

Theorem 3. The estimator $\hat{k}_{T}$ of $k$ admits the asymptotic normality, i.e.

$$
\frac{\left(\hat{k}_{T}-k\right) \sqrt{T}}{\sigma} \stackrel{L}{\rightarrow} N(0,1) \quad \text { a.s. }[R] \text { as } T \rightarrow \infty,
$$

where $L$ is the convergence in distribution.

Proof: According to the above mentioned, we can have the fact

$$
\begin{aligned}
& \ell_{k}^{\prime}(k, \alpha) \\
= & p R_{T}-p^{2} k T+\alpha p^{2} \int_{0}^{T} H\left(R_{t}\right) d t \\
= & p\left[\int_{0}^{T} p\left(k-\alpha H\left(R_{t}\right)\right) d t+\sigma p d W_{t}\right]-p^{2} k T \\
& +\alpha p^{2} \int_{0}^{T} H\left(R_{t}\right) d t \\
= & \sigma p^{2} W_{T}
\end{aligned}
$$

and

$$
\ell_{k}^{\prime \prime}(k, \alpha)=-p^{2} T \text {. }
$$

On the other hand, through the taylor expansion, we can get

$$
\ell_{k}^{\prime}(k, \alpha)
$$$$
=\ell_{k}^{\prime}\left(\hat{k}_{T}, \alpha\right)+\left(k-\hat{k}_{T}\right) \ell_{k}^{\prime \prime}\left(\hat{k}_{T}+\eta\left(k-\hat{k}_{T}\right), \alpha\right)
$$$$
=p^{2} T\left(\hat{k}_{T}-k\right) \text {, }
$$

where $|\eta| \leq 1$. It is easy to see that

$E\left[\ell_{k}^{\prime}(k, \alpha)\right]=E\left[\sigma p^{2} W_{T}\right]=0$,

and

$$
\operatorname{Var}\left(\ell_{k}^{\prime}(k, \alpha)\right)
$$$$
=E\left[\ell_{k}^{\prime}(k, \alpha)-E\left[\ell_{k}^{\prime}(k, \alpha)\right]\right]^{2}
$$

$=E\left[\ell_{k}^{\prime}(k, \alpha)\right]^{2}$

$=E\left[\sigma p^{2} W_{T}\right]^{2}$

$=\sigma^{2} p^{4} T$.

\section{Volume 5 Issue 6, June 2016}




\title{
International Journal of Science and Research (IJSR) \\ ISSN (Online): 2319-7064
}

Index Copernicus Value (2013): 6.14 | Impact Factor (2015): 6.391

By central limit theorem(see Prakasa Rao [12], Theorem B.10, page 313), we have the conclusion,

$$
\begin{aligned}
& \frac{\ell_{k}^{\prime}(k, \alpha)-E\left[\ell_{k}^{\prime}(k, \alpha)\right]}{\sqrt{\operatorname{Var}\left(\ell_{k}^{\prime}(k, \alpha)\right)}} \\
&= \frac{\ell_{k}^{\prime}(k, \alpha)}{\sqrt{\sigma^{2} p^{4} T}} \\
&= \frac{\ell_{k}^{\prime}(k, \alpha)}{\sigma p^{2} \sqrt{T}} \\
& \stackrel{L}{\longrightarrow} N(0,1) \text { as } T \rightarrow \infty .
\end{aligned}
$$

Combing with

$$
\ell_{k}^{\prime}(k, \alpha)=p^{2} T\left(\hat{k}_{T}-k\right),
$$

Hence, we can proof the conclusion.

$\frac{p^{2} T\left(\hat{k}_{T}-k\right)}{\sigma p^{2} \sqrt{T}}=\frac{\left(\hat{k}_{T}-k\right) \sqrt{T}}{\sigma} \stackrel{L}{\longrightarrow} N(0,1)$

$T \rightarrow \infty$.

Theorem 4. The estimator $\hat{\alpha}_{T}$ of $\alpha$ admits the asymptotic normality, i.e.

$$
\frac{\left(\hat{\alpha}_{T}-\alpha\right) \sqrt{\int_{0}^{T}\left(H\left(R_{t}\right)\right)^{2} d t}}{\sigma} \stackrel{L}{\rightarrow} N(0,1)
$$

a.s.[R] as $T \rightarrow \infty$,

where $L$ is the convergence in distribution.

Proof: Similarly to the proof of the Theorem 3, we can have that

$$
\begin{aligned}
& \ell_{\alpha}^{\prime}(k, \alpha) \\
= & -p \int_{0}^{T} H\left(R_{t}\right) d R_{t}+k p^{2} \int_{0}^{T} H\left(R_{t}\right) d t \\
& -\alpha p^{2} \int_{0}^{T}\left(H\left(R_{t}\right)\right)^{2} d t \\
= & -p \int_{0}^{T} H\left(R_{t}\right)\left[p\left(k-\alpha H\left(R_{t}\right)\right) d t+\sigma p d W_{t}\right] \\
& +k p^{2} \int_{0}^{T} H\left(R_{t}\right) d t-\alpha p^{2} \int_{0}^{T}\left(H\left(R_{t}\right)\right)^{2} d t \\
= & -p^{2} k \int_{0}^{T} H\left(R_{t}\right) d t+\alpha p^{2} \int_{0}^{T}\left(H\left(R_{t}\right)\right)^{2} d t \\
& -\sigma p^{2} \int_{0}^{T} H\left(R_{t}\right) d W_{t}+k p^{2} \int_{0}^{T} H\left(R_{t}\right) d t \\
& -\alpha p^{2} \int_{0}^{T} H^{2}\left(R_{t}\right) d t \\
= & -\sigma p^{2} \int_{0}^{T} H\left(R_{t}\right) d W_{t}
\end{aligned}
$$

$\ell^{\prime \prime} \alpha(k, \alpha)=-\sigma p^{2} \int_{0}^{T}\left(H\left(R_{t}\right)\right)^{2} d t$

On the other hand, through the taylor expansion, we can get

$$
\begin{aligned}
& \ell_{\alpha}^{\prime}(k, \alpha) \\
= & \ell_{\alpha}^{\prime}\left(k, \hat{\alpha}_{T}\right)+\left(\alpha-\hat{\alpha}_{T}\right) \ell_{\alpha}^{\prime \prime}\left(k, \hat{\alpha}_{T}+\xi\left(\alpha-\hat{\alpha}_{T}\right)\right) \\
= & p^{2}\left(\hat{\alpha}_{T}-\alpha\right) \int_{0}^{T}\left(H\left(R_{t}\right)\right) d t .
\end{aligned}
$$

where $|\xi| \leq 1$. It is easy to see that

$$
E\left[\ell_{\alpha}^{\prime}(k, \alpha)\right]=E\left[-\sigma p^{2} \int_{0}^{T} H\left(R_{t}\right) d W_{t}\right]=0,
$$

and

$$
\begin{aligned}
& \operatorname{Var}\left(\ell_{\alpha}^{\prime}(k, \alpha)\right) \\
= & E\left[\ell_{\alpha}^{\prime}(k, \alpha)-E\left[\ell_{\alpha}^{\prime}(k, \alpha)\right]\right]^{2} \\
= & E\left[\ell_{\alpha}^{\prime}(k, \alpha)\right]^{2} \\
= & E\left[-\sigma p^{2} \int_{0}^{T} H\left(R_{t}\right) d W_{t}\right]^{2}
\end{aligned}
$$

$=\sigma^{2} p^{4} \int_{0}^{T}\left(H\left(R_{t}\right)\right)^{2} d t$.

By central limit theorem(see Prakasa Rao [12], Theorem B.10, page 313 ), we have the conclusion,

$$
\begin{aligned}
& \frac{\ell_{\alpha}^{\prime}(k, \alpha)-E\left[\ell_{\alpha}^{\prime}(k, \alpha)\right]}{\sqrt{\operatorname{Var}\left(\ell_{\alpha}^{\prime}(k, \alpha)\right)}} \\
&= \frac{\ell_{\alpha}^{\prime}(k, \alpha)}{\sqrt{\sigma^{2} p^{4} \int_{0}^{T}\left(H\left(R_{t}\right)\right)^{2} d t}} \\
&= \frac{\ell_{\alpha}^{\prime}(k, \alpha)}{\sigma p^{2} \sqrt{\int_{0}^{T}\left(H\left(R_{t}\right)\right)^{2} d t}} \\
& \stackrel{L}{\longrightarrow} N(0,1) \text { as } T \rightarrow \infty .
\end{aligned}
$$

Combing with

$$
\ell_{\alpha}^{\prime}(k, \alpha)=p^{2}\left(\hat{\alpha}_{T}-\alpha\right) \int_{0}^{T}\left(H\left(R_{t}\right)\right)^{2} d t,
$$

Hence, we can proof the conclusion.

$$
\begin{gathered}
\frac{p^{2}\left(\hat{\alpha}_{T}-\alpha\right) \int_{0}^{T}\left(H\left(R_{t}\right)\right) d t}{\sigma p^{2} \sqrt{\int_{0}^{T}\left(H\left(R_{t}\right)\right) d t}} \\
=\frac{\left(\hat{\alpha}_{T}-\alpha\right) \sqrt{\int_{0}^{T}\left(H\left(R_{t}\right)\right) d t}}{\sigma} \\
\stackrel{L}{\longrightarrow} N(0,1) \text { as } T \rightarrow \infty .
\end{gathered}
$$

At last, we show that the parameter estimation for skew Ornstein-Uhlenbeck when $x>0$. That is to say, $d R_{t}=(1-p)\left(k-\alpha H\left(R_{t}\right)\right) d t+\sigma(1-p) d W_{t}, \quad x>0$.

\section{Volume 5 Issue 6, June 2016}

\author{
www.ijsr.net
}




\section{International Journal of Science and Research (IJSR) \\ ISSN (Online): 2319-7064}

Index Copernicus Value (2013): 6.14 | Impact Factor (2015): 6.391

Similarly to the conclusion mentioned above, we can derive the estimators of the drift parameter:

$$
\left\{\begin{array}{l}
\hat{k}_{T}=\frac{\int_{0}^{T} H\left(R_{t}\right) d R_{t} \int_{0}^{T} H\left(R_{t}\right) d t-R_{T} \int_{0}^{T}\left(H\left(R_{t}\right)\right)^{2} d t}{(1-p)\left[\left(\int_{0}^{T} H\left(R_{t}\right) d t\right)^{2}-T \int_{0}^{T}\left(H\left(R_{t}\right)\right)^{2} d t\right]}, \\
\hat{\alpha}_{T}=\frac{T \int_{0}^{T} H\left(R_{t}\right) d R_{t}-R_{T} \int_{0}^{T} H\left(R_{t}\right) d t}{(1-p)\left[\left(\int_{0}^{T} H\left(R_{t}\right) d t\right)^{2}-T \int_{0}^{T}\left(H\left(R_{t}\right)\right)^{2} d t\right]} .
\end{array}\right.
$$

Theorem 5. The estimator $\hat{k}_{T}$ of $k$ admits the strong consistency, i.e.

$\hat{k}_{T} \rightarrow k \quad$ a.s. $[R]$ as $T \rightarrow \infty$.

Theorem 6. The estimator $\hat{\alpha}_{T}$ of $\alpha$ admits the strong consistency, i.e.

$\hat{\alpha}_{T} \rightarrow \alpha \quad$ a.s. $[R]$ as $T \rightarrow \infty$.

Theorem 7. The estimator $\hat{k}_{T}$ of $k$ admits the asymptotic normality, i.e.

$$
\frac{\left(\hat{k}_{T}-k\right) \sqrt{T}}{\sigma} \stackrel{L}{\rightarrow} N(0,1) \text { a.s. }[R] \text { as } T \rightarrow \infty,
$$

where $L$ is the convergence in distribution.

Theorem 8 . The estimator $\hat{\alpha}_{T}$ of $\alpha$ admits the asymptotic normality, i.e.

$$
\frac{\left(\hat{\alpha}_{T}-\alpha\right) \sqrt{\int_{0}^{T}\left(H\left(R_{t}\right)\right)^{2} d t}}{\sigma} \rightarrow N(0,1)
$$

a.s. $[R]$ as $T \rightarrow \infty$,

where $L$ is the convergence in distribution.

Similary to the above proof, so we omit.

\section{References}

[1] L. Bo, Y. Wang, X. Yang and G. Zhang "Maximum likelihood estimation for reflected Ornstein-Uhlenbeck processes," Journal of Statistical Planning and Inference, pp. 588-596, 2011.

[2] L. Bo and X. Yang "Sequential maximum likelihood estimation for reflected generalized OrnsteinUhlenbeck processes," Statistics and Probability Letters, pp. 1374-1382, 2012.

[3] R. S. Cantrell and C. Cosner "Diffusion models for population dynamics incorporating individual behavior at boundaries: appliations to refuge design," Theoretical Population Biology, pp. 189-207, 1999.

[4] M. Decamps, A. D. Schepper and M. Goovaerts "Applications of delta-function perturbation to the pricing of derivative securities," Physica a-statistical mechanics and its applications, pp. 677-692, 2004.

[5] Y. Hu, C. Lee, M. Lee and J. Song " Parameter estimation for reflected Ornstein-Uhlenbeck processes with discrete observations," Statistical Inference for Stochastic Processes, pp. 1-13, 2014.

[6] K. Itô and H. P. McKean "Diffusion Processes and their Sample Paths,” Springer Verlag, 1974.
[7] R. S. Liptser and A. N. Shiryayev "Statistics of Random Processes:Applications,” Springer, New York, 1978.

[8] R. Lang "Effective conducitivity and skew Brownian motion," Journal if statistical physics, pp. 125-146, 1995.

[9] A. Lejay " On the constructions of the skew Brownian motion,” Probability Surveys, pp. 413-466, 2006.

[10] C. Lee, J. P. N. Bishwal, M. H. Lee “ Sequential maximum likelihood estimation for reflected Ornstein-Uhlenbeck processes," Journal of Statistical Planning \& Inference, pp. 1234-1242, 2012.

[11] P. Protter " Stochastic integration and differential equations,” Springer, 1990.

[12] B. L. S. Prakasa Rao " Statistical inference for diffusion type processes," Co-published by Oxford University Press, New York, 1999.

[13] S. Wang, S. Song and Y. Wang “ Skew Ornstein-Uhlenbeck processes and their financial applications," Journal of Computational and Applied Mathematics, pp. 363-382, 2015. 